\title{
Applications géomatiques adaptées à la gestion de l'érosion littorale
}

\author{
Bernard Long ${ }^{(\mathrm{a})^{*}}$, Régis Xhardé( $\mathrm{a}^{* *}$, Maude Boucher ${ }^{(\mathrm{a})^{* *}}$, \\ Vincent Tao $^{(\mathrm{b})}$, et Donald Forbes ${ }^{(\mathrm{c})}$, \\ ${ }^{(\mathrm{a}) *}$ professeur, ${ }^{(\mathrm{a}) * *}$ étudiants, INRS-ETE, Québec \\ (b) professeur, (York University, Toronto) \\ ${ }^{(c)}$ chercheur, (GSC-Atlantic, Halifax)
}

\section{Résumé}

L’objectif de ce projet est de fusionner les résultats de deux techniques et de transférer les connaissances vers des applications orientées pour la gestion des littoraux. Au cours de deux relevés, les données de LIDAR et de vidéogrammétrie ont été acquis le long d'un couloir de $120 \mathrm{~km}$ de côte. En même temps un programme de relevé au sol a été entrepris pour déterminer les paramètres géomatiques, sédimentologiques, géologiques et géotechniques sur 8 station pour étalonner le signal de retour du LIDAR. Pour analyser les données, le groupe est engagé dans l'analyse et la visualisation des données LIDAR en développant un algorithme hiérarchisant les données de terrain pour générer des modèles numériques de terrain à partir des signaux de retour du LIDAR. Durant la deuxième phase, les systèmes SHOALS et multi-faisceaux seront testés.

\section{$\underline{\text { Abstract }}$}

The objective of this project is to merge together the results of two techniques and transfer the knowledge to application oriented beach management users. During two surveys, LIDAR and airborne videogrammetry data has been acquired along a $120 \mathrm{~km}$ corridor. At the same time, one land program was initiate to measure several geomatical, sedimentological, geological and geotechnical parameters in 8 stations to calibrate the return signal intensity of the LIDAR. To process the data, the group has been extensively engaged both in lidar data processing and analysis and visualization. We developed a hierarchical terrain recovery algorithm to generate digital terrain models (DTMs) from single and multiple returns lidar data. During the second phase of the project SHOALS and multibeams system will be tested.

Mots-clés: Érosion côtière, LIDAR, Vidéogrammétrie, SHOALS, Multi-faisceaux

\section{Introduction}

La gestion de l'érosion marine devient chaque jour plus complexe en raison de la pression anthropique qui s'intensifie et se diversifie sur le milieu naturel. La remontée du niveau marin constatée récemment vient compliquer cette gestion aussi est-il impératif de mettre en œuvre des méthodes qui permettent d'étudier avec précision l'ensemble du domaine littoral. Les applications des méthodes géomatiques deviennent primordiales pour entreprendre ce travail.

Le projet présenté se veut une réponse à ce problème, il est conçu en deux phases, une première qui ne concerne que le milieu terrestre et une deuxième qui intègre l'ensemble de la zone côtière entre la limite d'action des houles au large et l'arrière plage. Il a pour but de développer une nouvelle classe de fusion de données et de méthodes d'intégration applicables à la gestion du littoral le long de corridors spécifiques de relevés. 
Il doit permettre d'accélérer les transferts technologiques entre les industries de l'environnement et des ressources, des agences gouvernementales et des universités pour mettre en œuvre une approche géomatique à travers une recherche multidisciplinaire et multisectorielle en utilisant des équipes multi-partenaires.

Ce projet doit déterminer la vulnérabilité au changement climatique et à l'érosion côtière, des infrastructures situées le long de la baie des Chaleurs dans l'Est du Canada. Plusieurs outils aéroportés sont utilisés simultanément soit dans la première phase, la vidéogrammétrie, le LIDAR et dans la deuxième phase le SHOALS et les sondeurs multi-faisceaux. Une série de relevés est entreprise au sol, pour étalonner ces mesures aéroportées et marines.

\section{$\underline{\text { 2.Site d'études }}$}

\subsection{Physiographie du site:}

La zone étudiée est constituée d’une série de sites qui représentent la diversité des environnements rencontrés dans l'Est du Canada. Les sites d'études sont répartis le long de la rive Nord de la baie des Chaleurs, dans la partie ouest du golfe du Saint-Laurent. Cette baie s'étire sur $180 \mathrm{~km}$ et sa largeur varie de 25 à $38 \mathrm{~km}$. Les deux sites les plus orientaux sont situés à l'extérieur de la baie et sont ouverts aux houles du golfe du Saint-Laurent.

La partie interne de la baie est contrôlée par les courants de marée et par des clapots locaux alors que les sites orientaux sont soumis à des houles pouvant atteindre $11 \mathrm{~s}$ de période et plus de 4,5 m de hauteur significative. L'amplitude de la marée varie en vive eau d'est en ouest de 1,6 m à l'est, à 2,8 $\mathrm{m}$ au centre et 2,25 $\mathrm{m}$ à l'ouest et en morte eau de respectivement $0,7 \mathrm{~m}, 0,9 \mathrm{~m}$ et $0,5 \mathrm{~m}$. Les courants de marée associés peuvent atteindre entre 40 et $60 \mathrm{~cm} / \mathrm{s}$ en jusant et $20 \mathrm{~cm} / \mathrm{s}$ en flot dans la partie interne de la baie (site de Maria). Ils sont inférieurs sur les autres sites.

Le rivage est bordé de plaines quaternaires (systèmes deltaïques fluvio-glaciaires constitués de dépôts de sables, de graviers et galets ou prodeltaïques constitués d'argiles et silts marins), de falaises formées de roches carbonifères (grès, conglomérats et pélites) et dévoniennes (calcaires). Ces falaises sont actuellement en érosion et alimentent à divers degrés un transport littoral qui se caractérise, localement par la formation de flèches (Paspébiac) ou cordon sédimentaires (Barachois, Bonaventure). L’apport des rivières est pratiquement inexistant et les estuaires sont sous une influence mixte de houles et des crues printanières.

\subsection{L'érosion actuelle :}

Depuis plusieurs années, le littoral gaspésien et les infrastructures côtières qui s’y rattachent, sont menacés par l'érosion littorale. La route principale passe en plusieurs points à la limite de la haute plage; elle a dû être déplacée une fois et elle est à nouveau menacée.

Une étude d'envergure a été entreprise sur différents sites types du littoral de la Baie de Chaleurs dans le but de caractériser le type d'érosion relatif aux environnements sédimentaires, géologiques et hydrodynamiques et d'y proposer des solutions à moyen et long terme adaptées à ces environnements.

Les plages étudiées sont des plages de faciès mixte (sable, graviers et galets) dont les stocks sédimentaires sont faibles sur toute la zone littorale (du haut de plage à la zone de fermeture) et dont l'arrière-plage est généralement déconnectée du système. 


\section{Le projet de recherche:}

Le travail de terrain fait suite à un programme de relevés topographiques effectués suivant une approche classique (théodolite) sur les sites de Maria, Saint-Siméon /Bonaventure et CapEspoir/Anse-à-Beaufils qui avait mis en évidence des érosions dans le profil de 10 à 20 $\mathrm{m}^{3} / \mathrm{m} / \mathrm{an}$ et des engraissements de certaines cellules de 0,5 à $7 \mathrm{~m}^{3} / \mathrm{m} / \mathrm{an}$. Les relevés sismiques effectués sur l'avant-plage montrent que cette dernière est généralement dépourvue de sédiments remobilisables et contribue très peu à l'équilibre de la plage.

La mission de relevés aéroportés (LIDAR et vidéogrammétrie) associés à des mesures de terrain a été entreprise en mai 2003 et mai 2004. Les stations d'étalonnages sont géoréférencées et les prélèvements de surface ont pour but d'analyser les paramètres qui influencent l'intensité du signal de retour, soit: la granulométrie, la teneur en eau, les paramètres géotechniques (pénétromètres et scissomètres) et les couleurs. Onze sites sont analysés : Malbaie, Barachois, Percé, Anse-à-Beaufils, Cap Espoir, Pabos, Saint-Godefroi, Paspébiac, Bonaventure, Saint-Siméon et Maria, soit un total de 120 km de côtes. Les mêmes sites seront couverts lors des relevés aéroportés utilisant le système SHOALS.

\section{Méthodologie}

\subsection{La vidéogrammétrie}

La vidéogrammétrie est une technique de mesure qui permet en deux survols consécutifs, de capter des images verticales et obliques. Le traitement des données utilise la méthode de la stéréoréstitution numérique. La caméra numérique (Sony DFW-SX-900) capte les images de la surface terrestre dans le domaine du visible du spectre électromagnétique.

\subsubsection{L'équipement}

Les données de base sont géoréférencées ( $\mathrm{x}$, y et z), l'exploitation est assurée par le logiciel Cyclop-3D et le traitement par le logiciel KRONOS. La caméra numérique (Sony DFW-SX-900) acquière des données à raison de 5 images/seconde, la largeur du corridor de relevé est de $400 \mathrm{~m}$ et la résolution spatiale de $0,32 \mathrm{~m}$. Le recouvrement longitudinale (R) est de $80 \%$ tant en couverture stéréo verticale qu'en couverture oblique ${ }^{[1]}$. Les traitements de données sont basées sur le système CYCLOP-3D et la caméra numérique sur l'utilisation du logiciel d'acquisition KRONOS. Cette caméra peut produire trois formats soit : le DV (720 x 480 pixels), le XGA (1 024 x 768 pixels) ou le SXGA (1 280 x 960 pixels). Le système d'acquisition est complété par un système de positionnement (récepteur GPS), un microordinateur portable (Pentium 4, 1.6GHz ou plus rapide) et le système d'alimentation.

\subsubsection{Traitement d'image vidéographique}

Pour les besoins du projet GEOIDE, la prise d'imagerie numérique est simultanée au balayage laser LIDAR. L'altitude de vol est de $800 \mathrm{~m}$, ce qui permet de suivre un couloir de $400 \mathrm{~m}$ au sol avec une résolution spatiale (pixel) de $30 \mathrm{~cm}$ en vertical et $0,16 \mathrm{~m}$ en oblique. Un étalonnage est effectuée avant le vol pour déterminer l'orientation intérieure, en épurant les erreurs de distorsions des lentilles, de la courbure de la terre et de la réfraction atmosphérique. L'orientation externe est effectuée en post-traitement, soit à partir des équations phogrammétriques, soit à partir des coordonnées-terrain d'un point. Ces derniers sont établis à partir de la mesure des coordonnées d'un point sur deux images et de quelques points de références sur le terrain. Dans ce dernier cas, deux corrections sont effectuées :

- L'orientation relative pour positionner les paires d'images (couple stéréoscopique) et reconstituer la position des images au moment de la prise de vue. 
- L'orientation absolue pour transformer les coordonnées-modèle calculées en coordonnées terrain. La précision devient alors de $30 \mathrm{~cm}$.

Par la suite, à l'aide du logiciel vidéo KRONOS, les images sont géo-référencées en associant en temps réel, les images en format numérique (AVI, DV, XGA ou SXGA) aux coordonnées géographiques issues de données GPS et inertielles. Puis, le logiciel Cyclop-3D permet l'évaluation d'impacts environnementaux à partir de deux relevés effectués à des dates différentes. Ce programme détermine des surfaces et des volumes. Les images verticales sont utilisées pour cartographier et mesurer des objets alors que les images obliques servent à visualiser la stratigraphie, définir les processus d'érosion et à réaliser l'inventaire de cicatrices de glissements.

\subsection{Le LIDAR (Light Detection And Ranging)}

\subsubsection{Le principe}

Le système LIDAR est basé sur le principe des systèmes RADAR: des impulsions de très courte durée (nanoseconde) de longueur d'onde proche de l'infrarouge sont émises par un laser. Le temps mis entre l'émission de l'onde et son écho correspond au double de la distance parcourue.

\subsubsection{L'équipement}

Les relevés LIDAR sont effectués en marée basse de vive eau, au moyen d'un système laser ALTM-2050 d'Optech utilisant un laser infrarouge de longueur d'onde de $1064 \mathrm{~nm}$. La fréquence d'émission est de $50 \mathrm{kHz}$ et la fréquence de balayage est de $17 \mathrm{~Hz}$. Les données de l'intensité du signal de retour des premiers et derniers échos sont enregistrées simultanément aux données de GPS (Trimble 4000 SSI). Les données de positionnement sont corrigées en post-traitement a l'aide des stations fixes de la Garde Côtière. L'altitude de la plate-forme d'acquisition (un Navaro) est déterminée grâce à une centrale inertielle Applanix fonctionnant à $200 \mathrm{~Hz}$ et qui possède une précision de $0,005^{\circ}$ pour le roulis et le tangage et de $0,015^{\circ}$ pour le cap. L'avion vole à une altitude de $800 \mathrm{~m}$ et couvre un corridor de $400 \mathrm{~m}$. L'espacement des points au sol est de $30 \mathrm{~cm}$ le long d'un balayage et de $150 \mathrm{~cm}$ entre deux lignes de balayage. Lors de la première campagne de relevés un seul passage a été réalisé alors que durant la deuxième campagne un passage a été réalisé sur la même ligne de vol et un autre à $200 \mathrm{~m}$ plus au large afin de couvrir les falaises sous un angle oblique. De plus des lignes perpendiculaires au trait de côte ont permis de déterminer le coefficient de dispersion latéral du signal afin d’étalonner les intensités du signal de retour.

\subsubsection{Analyse des données d'altimétrie}

Le post-traitement des données consiste à améliorer la précision des positionnements obtenus par GPS et de classer les points LIDAR en deux catégories, ceux qui représentent le sol et ceux qui représentent le haut du couvert végétal.

Par la suite, a l'aide du logiciel ArcGIS 8.3, trois produits sont réalisés: les TIN (Triangulated Irregular Network ou Réseau irrégulier de triangulation), les MNT (Modèles Numériques de Terrain) et les MNI (Modèles Numériques d'Intensité). Par la suite, trois autres produits sont développés soit les cartes topographiques en courbes de niveaux, les modèles numériques de pentes (MNP) et les modèles numériques d'orientation de pentes (MNOP). La superposition de deux MNT permet de déterminer les modèles numériques d'érosion (MNE), d'établir des bilans sédimentaires et des modèles de prévision des risques (MPR). L’indice de risque est déterminé pour l'ensemble de la zone côtière en indexant une cote pour chaque élément morphologique représentatif (pente, forme des chenaux de ruissellements...) mesurés par fusion de MNT et des MNI. 


\subsubsection{Analyse de l'intensité du signal de retour}

Les MNI représentent les variations environnementales du milieu. Aussi, une analyse des paramètres géotechniques et stratigraphiques a été entreprise en analysant en laboratoire (mesures de l'intensité scanographique, de la teneur en eau, de la minéralogie et de la granulométrie des sédiments) des échantillons prélevés sur les plages. L’intensité LIDAR moyenne est calculée à partir des points situés dans un rayon de 1,6 mètres autour du point d'échantillonnage (entre 8 et 11 points). Parallèlement, des échantillons de roches provenant des falaises sont également analysés par scanographie. Les valeurs d'intensité tomographique (IT), le type de roche (grès, pelite, conglomérat et calcaire), la porosité et le degré d’altération du ciment (grès et conglomérat) sont également reliés à l'intensité moyenne du signal de retour du LIDAR.

\subsection{Le SHOALS (Scanning Hydrographic Operational Airborne)}

\subsubsection{Le principe:}

Le SHOALS est un système LIDAR qui utilise deux lasers. Le premier laser émet des impulsions proches de l'infrarouge (longueur d'onde de $1064 \mathrm{~nm}$ ) qui se réfléchissent à la surface de l'eau. Le deuxième laser émet des impulsions dans le domaine du vert (longueur d'onde de $532 \mathrm{~nm}$ ) qui pénètrent dans l'eau et se réfléchissent sur le fond. Ce dernier peut pénétrer jusqu'à $70 \mathrm{~m}$ dans des conditions exceptionnelles et généralement $50 \mathrm{~m}$ dans des conditions normales .

\subsubsection{L'équipement:}

La fréquence d'émission est de $1 \mathrm{kHz}$ et celle de balayage de $16 \mathrm{~Hz}$. L'angle de balayage est de $20^{\circ}$. La précision verticale est de $0,25 \mathrm{~cm}$ et la précision horizontale de $2,5 \mathrm{~m}$. La densité d'échantillonnage est variable ( $2 \times 2,3 \times 3,4 \times 4$ ou $5 \times 5 \mathrm{~m})$ Les données acquises sont les données altimétriques pour chacun des deux échos, les données d'intensité du signal de retour, les données de positionnement GPS et celles provenant de la centrale inertielle Applanix. L'avion vol à une altitude de 200 ou $400 \mathrm{~m}$ et couvre un corridor de 200 ou $400 \mathrm{~m}$.

Dans le présent projet, les données du système SHOALS sont comparées à celles provenant d'un système bathymétrique acoustique multi-faisceaux EM3000 qui émet à 300 $\mathrm{kHz}$ et qui a une ouverture angulaire de $120^{\circ}$, un espacement entre les faisceaux de $0,9^{\circ}$ au nadir et $1,8^{\circ}$ à $60^{\circ}$ et une ouverture du faisceau de $1,5^{\circ}$ au nadir et $3^{\circ}$ à $60^{\circ}$. La résolution est de $1,6 \%$ de la profondeur d'eau au nadir et $2,0 \%$ de la couche d'eau traversée à $60^{\circ}$.

\subsubsection{L'analyse du signal}

Les mêmes analyses que celles effectuées sur les données LIDAR sont utilisées pour l'analyse des données SHOALS tant au niveau des mesures de bathymétrie que des mesures d'intensité des signaux de retour. Une comparaison est effectuée avec les données de sondeurs multi-faisceaux afin de déterminer l’efficacité de chacune des deux méthodes.

\section{Résultats}

Durant les deux dernières années, deux survols de LIDAR ont été réalisés et par la suite, durant les deux années à venir, deux survols SHOALS sont planifiés.

\subsection{Les Modèles Numériques de Terrain (MNT)}

A partir des données du dernier retour filtré du signal LIDAR, des Modèles Numériques de Terrain (MNT) très précis ( \pm 0.25 mètres), ont été réalisées grâce au logiciel Arcview 8.3. pour chacune des zones. Un étalonnage entre les mesures de profil de plage établi par 
théodolite et par LIDAR a été effectué, la différence varie entre 39 et $0 \mathrm{~cm}$ ( Tableau 1) Un exemple des différents modèles de terrain est présenté (figure 1).

Les MNT permettent la détection de structures d'érosion de petite taille et la création de profils basés sur les données d'élévation LIDAR. La fiabilité des profils générés par ordinateur a été évaluée par comparaison avec des profils relevés sur le terrain.

Tableau 1: Tableau comparatif entre les mesures d'élévations obtenues par relevés LIDAR et par relevés théodolites le long d'un profil.

\begin{tabular}{|c|c|c|c|c|}
\hline points & description & Élévation terrain & $\begin{array}{c}\text { Élévation } \\
\text { LIDAR }\end{array}$ & Écart (Lid. -Ter.) \\
\hline 1 & Sommet falaise & $11,68 \mathrm{~m}$ & $11,70 \mathrm{~m}$ & $+0,02 \mathrm{~m}$ \\
\hline 2 & Début falaise & $11,55 \mathrm{~m}$ & $11,57 \mathrm{~m}$ & $+0,02 \mathrm{~m}$ \\
\hline 3 & Début glissement & $6,77 \mathrm{~m}$ & $7,00 \mathrm{~m}$ & $+0,23 \mathrm{~m}$ \\
\hline 4 & Fin glissement (haut) & $3,41 \mathrm{~m}$ & $3,80 \mathrm{~m}$ & $+0,39 \mathrm{~m}$ \\
\hline 5 & Fin glissement (bas) & $2,44 \mathrm{~m}$ & $2,20 \mathrm{~m}$ & $-0,24 \mathrm{~m}$ \\
\hline 6 & Creux d'avant-plage & $-0,50 \mathrm{~m}$ & $-1,15 \mathrm{~m}$ & $-0,60 \mathrm{~m}$ \\
\hline 7 & Crête d’avant-plage & $0,22 \mathrm{~m}$ & $-0,86 \mathrm{~m}$ & $-1,08 \mathrm{~m}$ \\
\hline pente & $\begin{array}{c}\text { Pente plage entre 5 } \\
\text { points et NMM }\end{array}$ & $\mathbf{8}^{\circ} 39^{\prime} 17^{\prime}$, & $8^{\circ} 51^{\prime} 28^{\prime} \pm 54^{\prime}$ & \\
\hline
\end{tabular}

Le long des falaises de Cap-Espoir, différentes actions d'érosion peuvent être distinguées: un type de profil vertical de la falaise associé à une érosion marine dominante et un profil en pente douce dans la partie supérieure de la falaise associé à une érosion climatique (figure 2). Des glissements de terrain et des marques d'érosion fluviatile sont mis en évidence aux MNT.

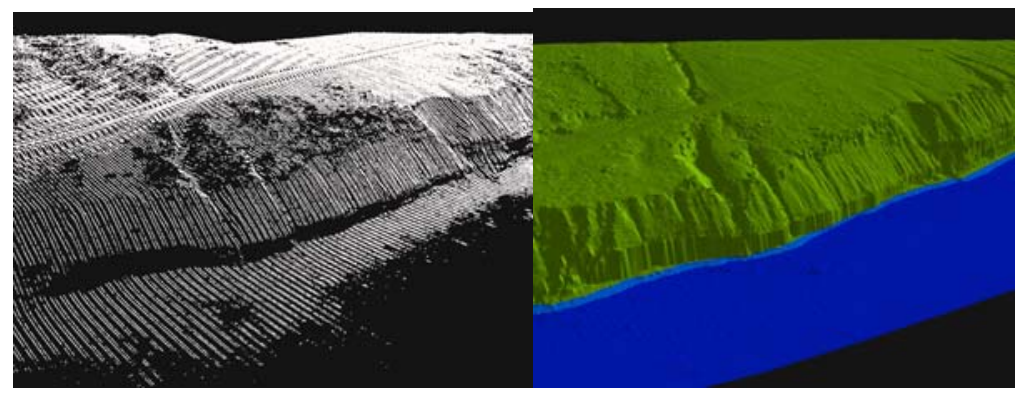

(1) Restitution des points altimétriques

(2) Modèle numérique de terrain (MNT)

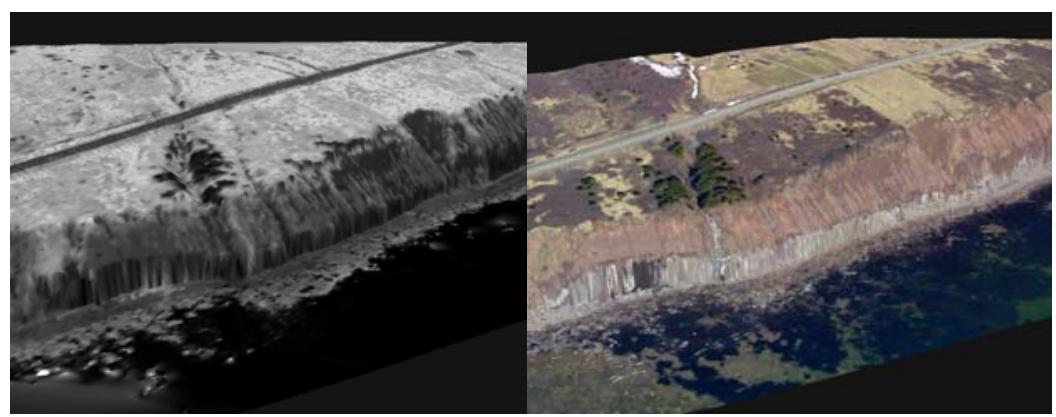

(3)Fusion entre un MNT et une image retour LIDAR

(4)Fusion entre une MNT et une image d'intensité du signal de vidéogrammétrique

Figure 1: Exemple des différentes fusions de données obtenues à partir des modèles numériques de terrain 


\subsection{Les mesures d'intensité du signal de retour}

L'étalonnage entre le signal d'intensité de retour du LIDAR et certains paramètres géotechniques ont été entrepris sur chaque type d'environnement (îles barrières, falaises érodées, plages et marais) et présentés tableau 2. De plus, une corrélation a été effectuée entre les valeurs d'intensité moyenne du signal de retour et le rapport de l'intensité tomographique sur la teneur en eau des sédiments (figure 3).

Parallèlement, un étalonnage est établi pour la roche en place. Le signal laser ne pénétre pas dans la roche, c’est l'intensité tomographique de la surface qui est considérée car elle reflète la densité de la couche d'altération de surface et non celle de la roche saine.

Tableau 2: Variation de l'intensité du signal de retour en fonction de l'environnement

\begin{tabular}{|l|l|c|c|}
\hline \multicolumn{1}{|c|}{ Environnement } & \multicolumn{1}{|c|}{ Matériel } & Intensité moyenne & Ecart-Type \\
\hline \hline \multirow{2}{*}{ Eau de mer } & Eau de mer & 7 & 6 \\
\hline Haut de plage & Sable (10-30\% teneur en eau) & 52 & 13,5 \\
\cline { 2 - 4 } & Sable + graviers (4-10\% teneur en eau) & 82 & 8,8 \\
\hline Arrière plage & Sable (<4\% teneur en eau) & 99 & 7,3 \\
\hline \multirow{2}{*}{ Pied de falaise } & Grés grossiers & 84,3 & 8,3 \\
& Conglomérats & 123,5 & 17,5 \\
\hline Sommet de falaises & Calcaires & 160 & 16,6 \\
\hline
\end{tabular}

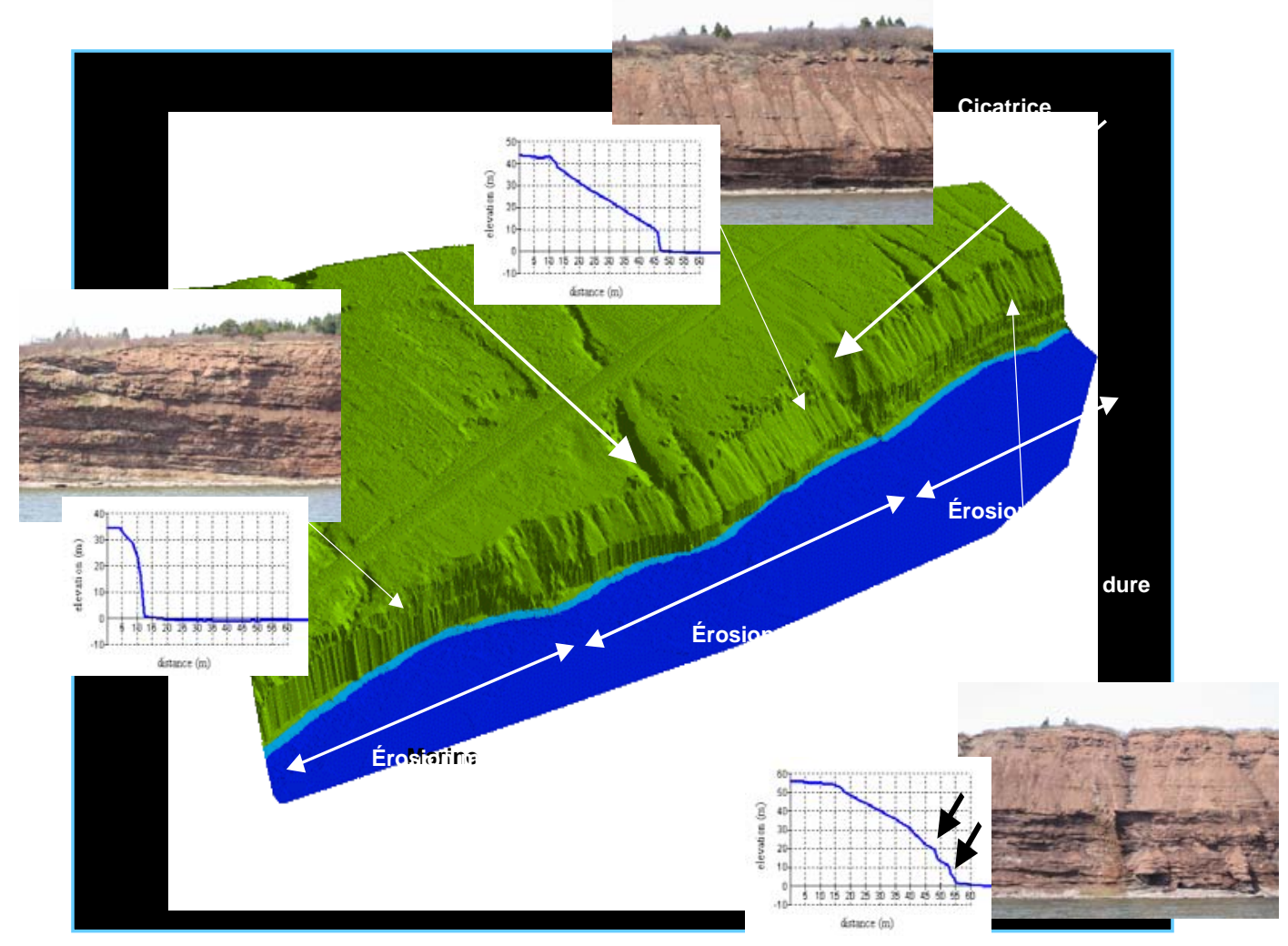

Figure 2: Détermination des unités géomorphologiques le long d'une cellule littorale. La position des lits de roche dure déterminent le type d'érosion de la falaise. 
La relation mathématique entre l'intensité moyenne du LIDAR et le rapport entre l'intensité tomographique et la teneur en eau est :

$\mathrm{I}_{\mathrm{L}}=\mathrm{C}_{1} * \mathrm{IT} /\left(\mathrm{C}_{2} *{ }_{\mathrm{W}}+\mathrm{IT}\right)$

où: - $\mathrm{I}_{\mathrm{L}}$ est l'intensité moyenne du signal de retour du LIDAR

- IT est l’intensité tomographique mesurée par scanographie

- $\mathrm{w}$ est la teneur en eau

- $\mathrm{C}_{1}$ et $\mathrm{C}_{2}$ sont des constantes

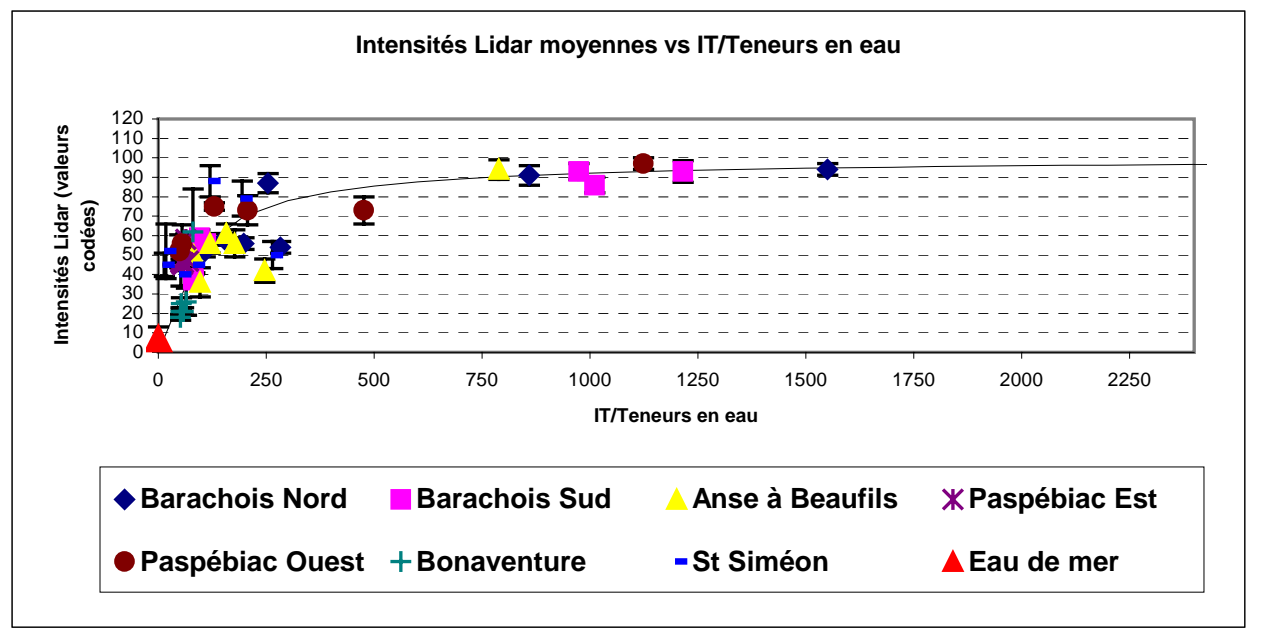

Figure 3: Relation de l'intensité du signal de retour avec les paramètres environnementaux.

\subsection{Les données vidéogrammétriques}

Les données vidéogrammétriques sont utilisées pour effectuer des mesures qualitatives car ce système ne permet pas encore d'intégrer les données de la centrale inertielle. Le logiciel MOSAIC 3D permet de construire des mosaïques qui doivent être géoréférencées à partir de points de contrôle sol. Mais ces mosaïques peuvent être superposées soit aux MNT (figure 1) soit aux MNI.

\subsection{Les données SHOALS}

L'utilisation du système SHOALS permet d'étendre l'acquisition des données au système infra littoral en intégrant la plage visible et la plage sous-marine (figure 4) à la pointe Norriego en Floride. En comparant deux relevés, une détermination des variations bathymétriques a été effectuée pour déterminer les volumes de sédiments à draguer ${ }^{[2]}$ ou les variations du profil de la zone côtière ${ }^{[3]}$. La comparaison de relevés SHOALS et multifaisceaux effectues sur un même site permet de déterminer la limite d'utilisation des deux techniques et d'étalonner les réponses des deux intensités du signal de retour. 


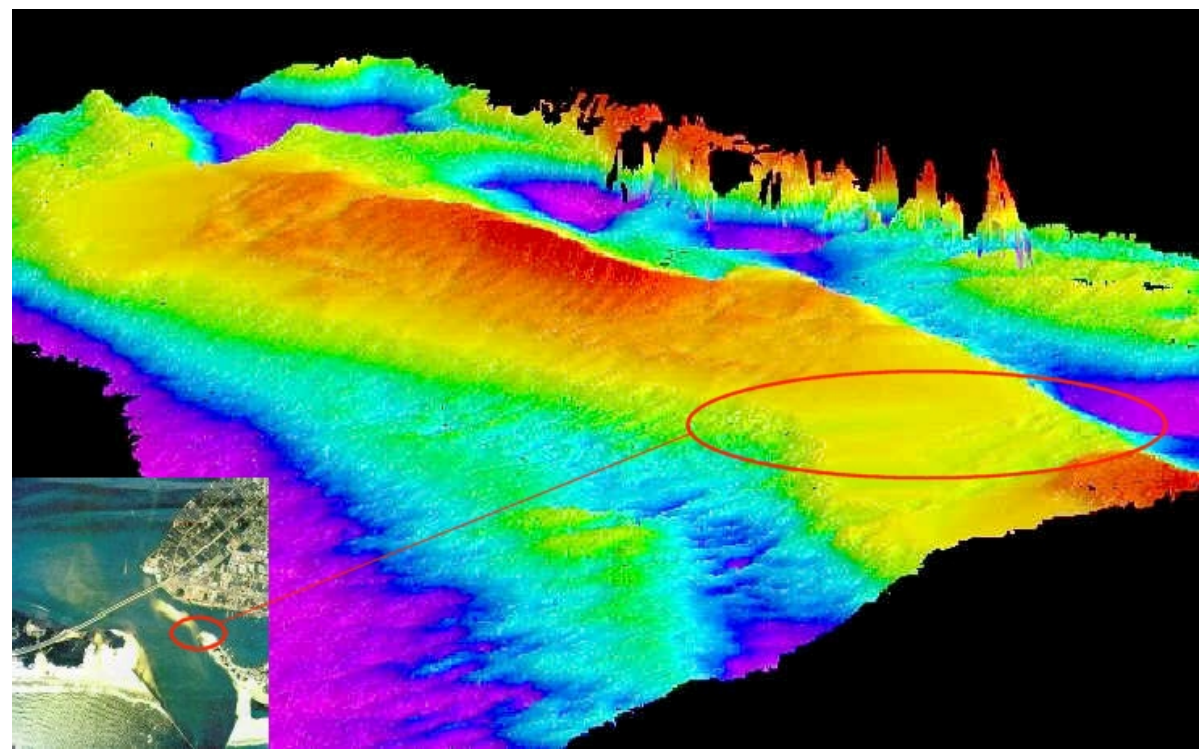

Figure 4: Exemple de relevé SHOALS à Pointe Norriego, Floride -Bathymétrie 3D effectuée avec le SHOALS

La rupture de la flèche sableuse de Norriego Point a été mesurée après le passage de l'ouragan Opal en 1995 (source: USACE - US Army Engineer District, Mobile. Joint Airborne Lidar Bathymetry Technical Center of Expertise (JALBTCX) - Mobile, Alabama, USA)

\section{$\underline{\text { 6.Discussion }}$}

L'acquisition des images vidéogrammétriques permet la saisie en continu de la scène observée.

Chaque séquence vidéo contient 5 images par seconde qui correspond à un recouvrement longitudinal de presque $100 \%$.

Les images sont enregistrées sous format numérique sans support analogique.

L'avènement de la vidéogrammétrie autorise le captage géoréférencé des données en vertical et en oblique.

La génération des mosaïques à partir d’image géoréférencées Cyclop-3D.

La production de modèles numériques de terrain et des cartes thématiques et topographiques.

Pour le suivi de l'érosion des côtes, l'imagerie verticale est le plus souvent insuffisante pour mettre en évidence et visualiser les différentes couches de strate dont à besoin le géomorphologue pour comprendre le phénomène d'érosion du système côtier. Dans ce sens il devient primordial de prendre de images obliques avec le choix de l'angle le plus approprié de la vue qui est généralement de 45 degrés ${ }^{[4][1]}$. Toute-fois, il faut signaler que les caméras numériques n’offrent pas la même précision que les caméras à film. Leur manque de précision est causé par les dimensions trop réduites des senseurs CCD, qui est de l'ordre de quelque centimètres carrés ce qui est petit par rapport en comparaison avec le film aérien de $23 * 23$ $\mathrm{cm}^{[5]}$.

\subsection{Apports de l'analyse des MNT}

A partir des données LIDAR du dernier retour filtré, des Modèles Numériques de Terrain (MNT) très précis ( \pm 0.25 mètres), des images d’intensité et des images d'élévations ont été réalisées grâce au logiciel Arcview 8.3. pour chacune des zones. Les MNT (tableau 2-II) permettent la détection de structures d'érosion de petite taille et la création de profils basés sur les données d'élévation LIDAR. 
La fiabilité des profils générés par ordinateur a été évaluée par comparaison avec des profils relevés sur le terrain. Le long des falaises de Percé, différentes actions d'érosion peuvent être distinguées: un type de profil vertical de la falaise peut être associé avec une érosion marine dominante tandis qu'un profil présentant une pente douce dans la partie supérieure de la falaise peut être associée à une érosion climatique intense de la partie meuble de la falaise. De plus les glissements de terrain et les marques d'érosion fluviatile peuvent également être détectées grâce aux MNT. Des mesures similaires ont déjà été effectuées en Oregon ${ }^{[6]}$ et sur les côtes de Caroline du Nord ${ }^{[7]}{ }^{\left[{ }^{8]}\right.}$. Ainsi, l'étude de l'érosion littorale ne considère plus le retrait de la côte comme un retrait linéaire constant, mais bien comme un retrait discret qui reflète les hétérogénéités des structures littorales.

\subsection{Apports de l'analyse du signal de retour}

Les premiers résultats d'analyse montrent que la teneur en eau diminue la réflexion du signal laser; en effet, les échantillons prélevés en bas de plage sont saturés en eau et leurs valeurs d'intensité LIDAR moyenne sont inférieures aux échantillons prélevés en haut de plage. De plus, l'intensité tomographique (valeur IT) est influencée par la granulométrie des sédiments, les sédiments grossiers donnant des valeurs IT supérieures que celles de sable moyen. Au niveau des roches, les conglomérats et les grès fins possèdent des intensités tomographiques similaires, la plus faible valeur s'explique par une plus grande porosité de la roche. Les pierres d'enrochement calcaire sont plus denses et ont une intensité tomographique plus grande.

Néanmoins, c’est la densité de la surface altérée de la roche qui déterminera la valeur de l'intensité du signal de retour du LIDAR. Une relation a été établie entre l'intensité moyenne du LIDAR, la teneur en eau et l'intensité au CAT-Scan des échantillons de sédiments de plage. Cette équation comporte encore beaucoup de facteurs non contrôlés et des analyses en laboratoires sont en cours pour déterminer l'influence de la granulométrie et de la minéralogie.Pour étalonner le signal de retour du laser sur les roches, des échantillons ont été prélevés sur les falaises et des analyses de la porosité, la densité et la couleur permettront d'établir cette relation.

\section{3.Établissement d'un outil de gestion des risques et de management de la zone côtière:}

La fusion des données provenant des différentes techniques aéroportées ouvre la voie a une connaissance intégrée de toute la bande côtière et devrait permettre de réaliser un outil de gestion intégré, multidisciplinaire et multi sectorielle en utilisant des équipes multipartenaires. La précision de ces techniques et la rapidité de mise en œuvre de cet outil permet d'envisager un contrôle total de la zone côtière en intégrant tant les données altimétriques que les données bathymétriques, morphologiques et biologiques (habitat des poissons) pour une gestion concertée des différentes agences gouvernementales.

\section{Conclusion}

La fusion des données provenant des deux méthodologies, vidéogrammétrie et LIDAR, crée un nouvel outil de gestion du littoral. Etant donnée la rapidité d'acquisition et la précision des mesures, ce nouvel outil constitue l'approche future de la surveillance de la zone côtière.

L'apport du système SHOALS renforcera la puissance de ce nouvel outil car il permettra d'intégrer durant le relevé, à la fois la partie émergée et la partie marine de la zone côtière. La vidéogrammétrie constitue un nouvel outil qualitatif d'acquisition des données à références spatiales pour la surveillance et le suivi spatio-temporel des systèmes côtiers fortement touchés par l'érosion du littoral durant ces dernières décennies. L’utilisation de la 
vidéogrammétrie combinée au GPS et à une unité inertielle permettra de raffiner la précision de l'imagerie. Le système LIDAR constitue un système exceptionnel pour la mesure de l'érosion côtière spécialement dans les secteurs littoraux ou l'érosion se fait de manière discrète.

Le long des falaises, il est recommande d'effectuer deux lignes parallèles de relevés afin d'augmenter la densité des mesures sur la paroi en érosion. Une relation existe entre les intensités du signal de retour du LIDAR et les paramètres environnementaux, un premier étalonnage montre que la teneur en eau est un paramètre essentiel mais d'autres paramètres influencent le signal.

L'utilisation de la mesure de densité scanographique est appropriée car elle permet d'effectuer une investigation sur une épaisseur semblable à celle qui influence le signal laser (quelques millimètres). Les premières cartes morphologiques constituent un premier pas vers la création d’un outil décisionnel de la gestion des zones côtière.

\section{Références}

1.Larouche, C., Laflamme, C., Lévesque, R., Geo-3D Inc., Canada \& Dr. Denis, R., Hydro-Québec, Canada (2002). A Georeferenced Aerial Videography Technology Applied to Erosion Monitoring. GIM International.

2.Wozencraft M. and Irish J.L., 2001. Airborne Lidar surveys and regional sediment management. $4^{\text {th }}$ EARSeL Workshop on Lidar Remote sensing of Land and Sea. EARSeL proceeding No.1, Paris. 11 p.

3.White S.A., Wang Y. 2002. Utilizing DEMs derived from LIDAR data to analyze morphologic change in North Carolina coastline. Remote sensing of Environment, 5809, pp. $1-9$.

4.Williams, I.M., Leach, J.H.J., Wadley, V., and Barker, B. (1998). Creation of models for the measurement of marine species using Along Track Video (ATV). Oceans '98, Proceedings Vol. 3, pp. 1797-1801, IEEE/OES, Piscataway, NJ.

5.Boulianne , M., Chapman, M. A., Blais, J. A. R. (1988). Assembling a PC-based image processing system : Some practical considerations, Archives de la société Internationale de photogrammétrie et de télédétection, commission VI, Kyoto, Japan, pp. 11-20.

6.Revell D.L., Komar P.D., and Sallenger Jr. A.H. 2002. An application of LIDAR to analyses of El Nino erosion in the Netarts littoral cell, Oregon. Journal of Coastal Research, vol. 18(4), pp.792-801.

7.Sallenger Jr. A. H., Krabill W, Swift R., and Brock J. 2001. Quantifying Hurricaninduced coastal chances using topographic LIDAR. Coastal Dynamic '01, American Society of Civil Engineers, Proceeding pp. 1007-1016.

8.Stockdon H., Sallenger Jr. A.H., List J.H., and Holman R.A. 2002. Estimation of shoreline position and change using airborne topographic LIDAR data. Journal of Coastal Research, vol. 18(3), pp.502-513. 